\title{
Low-energy Möller scattering in a Maxwell-Chern-Simons Lorentz-violating planar model
}

\author{
M. M. Ferreira Junior* \\ Universidade Federal do Maranhão (UFMA), \\ Departamento de Física, Campus Universitário do Bacanga, São \\ Luiz - MA, 65085-580 - Brazil.
}

June 15, 2018

\begin{abstract}
One starts from a planar Maxwell-Chern-Simons model endowed with a Lorentz-violating term. The Dirac sector is introduced exhibiting a Yukawa and a minimal coupling with the scalar scalar and the gauge fields, respectively. One then evaluates the electron-electron interaction as the Fourier transform of the Möller scattering amplitude carried out in the non-relativistic limit. In the case of a purely time-like background, the interaction potential can be exactly solved, exhibiting a typical massless behavior far from the origin. The scalar interaction potential is always attractive whereas the gauge intermediation may also present attraction even when considered in the presence of the centrifugal barrier and the $A^{2}$ term. Such a result is a strong indication that electron-electron bound states may appear in this theoretical framework.
\end{abstract}

\section{Introduction}

In the beginning 90's, the Möller scattering was adopted as a theoretical tool to investigate the possible formation of electron-electron bound states in the context of a Maxwell-Chern-Simons electrodynamics 11. According to this procedure, one starts from the scattering amplitude (carried out at tree-level) to obtain the electron-electron interaction potential (Born approximation). As a settled down result, it was observed that the potential may come out negative whenever the topological mass exceeds the electron mass $\left(s>m_{e}\right)$, condition which is particularly discouraging in relation to the possibility of applying this kind of model to some condensed matter systems, where one usually deals with low-energy excitations. The introduction of the Higgs sector, arising from the spontaneous symmetry breaking [2], has shown to be a theoretical factor able to provide a scalar attractive interaction. The overall potential, consisting in the sum of the gauge and scalar contributions, may then be negative independently of the condition $s>m_{e}$, a necessary premise for the formation of Cooper pairs in the context of low-energy systems.

In the latest years, Lorentz-violating theories have been in focus of intensive investigation [3], 4]. In a recent work, a planar Lorentz-violating electrodynamics [5] was derived from the dimensional reduction of a Maxwell electrodynamics supplemented with the Carroll-Field-Jackiw (CFJ) term [6]. The consistency of this model has already been analyzed, revealing a model globally stable, causal and unitary for both time- and spacelike backgrounds [5]. The fact that the unitarity is assured makes feasible, at principle, the consistent quantization of this model, which sets it up as a candidate to be applied to situations where the quantization of the modes is a real condition (such as some condensed matter phenomena). In a posterior investigation [7, the equations of motion (for the field strengths and potentials) corresponding to this planar model were determined and solved in the static regime. The results obtained differ from

*e-mail: manojr@ufma.br 
the solutions of a pure MCS electrodynamics by background-depending corrections, which amount to relevant qualitative modifications. Indeed, the solutions have exhibited a typical massless behavior (in the electric sector) for the case of a timelike background and anisotropic behavior for the case of a spacelike background. It was also reported the possibility of obtaining an attractive electron-electron interaction as a consequence of the existence of well region in the behavior of the scalar potential $\left(A_{0}\right)$.

Lorentz covariance is certainly an essential feature of any relativistic system, mainly for ensuring the equivalence between all inertial frames. Once Lorentz symmetry is broken, such equivalence is lost, and each inertial frame starts to notice a different physics. It is a well known fact that condensed matter systems (CMS) are not endowed with Lorentz covariance, but with Galileo one, which holds as a genuine symmetry in the domain of isotropic low-energy systems. Having in mind that a CMS may be addressed as the low-energy limit of a relativistic model, there follows a straightforward correspondence between the breakdown of Lorentz and Galileo symmetries, in the sense that a CMS with violation of Galileo symmetry may have as counterpart a relativistic system endowed with breaking of Lorentz covariance.

Theoretical planar models able to provide attractive $e^{-} e^{-}$interaction potentials are relevant in the sense they may constitute a suitable framework to address the condensation of Cooper pairs, a fundamental characteristic of superconducting systems. Another well defined feature of a planar high-Tc superconductor concerns the symmetry of the order parameter (standing for the Cooper pair), which is described in terms of a spatially anisotropic d-wave [8. A theoretical framework able to provide an anisotropic $e^{-} e^{-}$interaction is the first step to the achievement of anisotropy for the order parameter. This is exactly the expected result to be obtained in the case a pure spacelike background, where the $e^{-} e^{-}$scattering potential may be identified with the one evaluated in the context of a CMS endowed with a privileged direction in space. Therefore, once an anisotropic CMS constitutes an example where the breakdown of the Galileo symmetry takes place, such a system may be properly approached as the low-energy limit of a Lorentz-violating electrodynamics in the presence of a pure spacelike background.

Having as main motivation the results achieved in ref. [7], which show that a fixed background induces sensitive effects at classical solutions, in this work one investigates the tree-level behavior of two interacting fermions in the context of a Lorentz-violating electrodynamics. By determining of the $e^{-} e^{-}$ interaction potential, one can verify to what extent the properties reported in the classical static analysis 7] are preserved in the context a dynamic evaluation. One can also study the possibility of achieving an $e^{-} e^{-}$interaction endowed with two relevant features: attractiveness and anisotropy, relevant properties in superconducting systems. Hence, the purpose is to carry out the $e^{-} e^{-}$interaction potential, exhibiting and stressing the corrections induced by the fixed background on the pure Maxwell-Chern-Simons result. For that, one first introduces the Dirac sector to the planar Lorentz-violating gauge model derived in ref. [5]. Taking into account the guidelines set up in refs. 1], 2], one then proceeds to evaluate the Möller scattering amplitude from which one derives the $e^{-} e^{-}$interaction (according to the Born approximation). The potential here attained is composed by two contributions, a scalar and a gauge one, since the $e^{-} e^{-}$ interaction is mediated by the massless scalar and the massive gauge fields. The scalar potential, absent in the context of a pure MCS model, is always negative, and may lead to a global attractive interaction regardless the sign of the gauge contribution. In the case of the gauge potential, it presents backgrounddepending terms that imply qualitative modifications, such as the possibility of being attractive for some parameters range, even when considered in the presence of the centrifugal barrier and the low-energy $A^{2}-$ Pauli term. Both the scalar and gauge potential possess a logarithm dependence, which is compatible with a massless behavior far from the origin.

This paper is outlined as follows. In Sec. II, one briefly exhibits the reduced model derived in ref. [5], supplemented by the fermion field. In Sec. III, one presents the spinors which fulfill the two-dimensional Dirac equation and are used to evaluate the scattering amplitude associated with the Yukawa and the minimal interactions. In Sec. IV, the interaction potential stemming form the scalar and gauge sectors are carried out, and the results are discussed. In Sec.V, one presents the concluding remarks. 


\section{Planar Lorentz-violating model}

The starting point is the planar Lagrangian ${ }^{1}$ obtained from the dimensional reduction of the CFJ-Maxwell electrodynamics [5], which consists in a Maxwell-Chern-Simons electrodynamics coupled to a massless scalar field $(\varphi)$ and to a fixed background $\left(v^{\mu}\right)$ through a Lorentz-violating term. One then considers the additional presence of a fermion field $(\psi)$ minimally coupled to the gauge field $\left(A_{\mu}\right)$ at the same time that exhibits a typical Yukawa coupling to the scalar field $(\varphi)$ :

$$
\begin{aligned}
\mathcal{L}_{1+2} & =-\frac{1}{4} F_{\mu \nu} F^{\mu \nu}+\frac{s}{2} \epsilon_{\mu \nu k} A^{\mu} \partial^{\nu} A^{k}-\frac{1}{2} \partial_{\mu} \varphi \partial^{\mu} \varphi+\varphi \epsilon_{\mu \nu k} v^{\mu} \partial^{\nu} A^{k}-\frac{1}{2 \alpha}\left(\partial_{\mu} A^{\mu}\right)^{2} \\
& +\bar{\psi}\left(i \not D-m_{e}\right) \psi-y \varphi(\bar{\psi} \psi) .
\end{aligned}
$$

Here, the covariant derivative, $\not D \psi \equiv\left(\not \partial+i e_{3} \not\right) \psi$, states the minimal coupling, whereas the term $\varphi(\bar{\psi} \psi)$ reflects the Yukawa coupling. In ref. [5], the propagators of the scalar $(\varphi)$ and gauge $\left(A_{\mu}\right)$ fields were properly evaluated as it appears below:

$$
\begin{aligned}
&\left\langle A^{\mu}(k) A^{\nu}(k)\right\rangle=i\left\{-\frac{1}{k^{2}-s^{2}} \theta^{\mu \nu}-\frac{\alpha\left(k^{2}-s^{2}\right) \otimes(k)+s^{2}(v \cdot k)^{2}}{k^{2}\left(k^{2}-s^{2}\right) \otimes(k)} \omega^{\mu \nu}-\frac{s}{k^{2}\left(k^{2}-s^{2}\right)} S^{\mu \nu}\right. \\
&+\frac{s^{2}}{\left(k^{2}-s^{2}\right) \otimes(k)} \Lambda^{\mu \nu}-\frac{1}{\left(k^{2}-s^{2}\right) \otimes(k)} T^{\mu} T^{\nu}+\frac{s}{\left(k^{2}-s^{2}\right) \otimes(k)}\left[Q^{\mu \nu}-Q^{\nu \mu}\right] \\
&\left.+\frac{i s^{2}(v \cdot k)}{k^{2}\left(k^{2}-s^{2}\right) \otimes(k)}\left[\Sigma^{\mu \nu}+\Sigma^{\nu \mu}\right]-\frac{i s(v \cdot k)}{k^{2}\left(k^{2}-s^{2}\right) \otimes(k)}\left[\Phi^{\mu \nu}-\Phi^{\nu \mu}\right]\right\}, \\
&\langle\varphi \varphi\rangle=\frac{i}{\otimes(k)}\left[k^{2}-s^{2}\right]
\end{aligned}
$$

where: $\nabla(k)=\left[k^{4}-\left(s^{2}-v \cdot v\right) k^{2}-(v \cdot k)^{2}\right]$, and the 2-rank tensors are defined as follows:

$$
\begin{aligned}
\theta_{\mu \nu} & =\eta_{\mu \nu}-\omega_{\mu \nu}, \omega_{\mu \nu}=\partial_{\mu} \partial_{\nu} / \square, S_{\mu \nu}=\varepsilon_{\mu \kappa \nu} \partial^{\kappa}, Q_{\mu \nu}=v_{\mu} T_{\nu} \\
T_{\nu} & =S_{\mu \nu} v^{\mu}, \Lambda_{\mu \nu}=v_{\mu} v_{\nu}, \quad \Sigma_{\mu \nu}=v_{\mu} \partial_{\nu}, \Phi_{\mu \nu}=T_{\mu} \partial_{\nu} .
\end{aligned}
$$

\section{The Möller Scattering amplitude}

The two-particle interaction potential is given by the Fourier transform of the two-particle scattering amplitude in the low-energy limit (Born approximation). In the case of the nonrelativistic Möller scattering, one should consider only the t-channel (direct scattering) [12] even for indistinguishable electrons, since in this limit they recover the classical notion of trajectory. From eq. (11), there follow the Feynman rules for the interaction vertices: $V_{\psi \varphi \psi}=i y ; V_{\psi A \psi}=i e_{3} \gamma^{\mu}$, so that the $e^{-} e^{-}$scattering amplitude are written as:

$$
\begin{aligned}
-i \mathcal{M}_{\varphi} & =\bar{u}\left(p_{1}^{\prime}\right)(i y) u\left(p_{1}\right)[\langle\varphi \varphi\rangle] \bar{u}\left(p_{2}^{\prime}\right)(i y) u\left(p_{2}\right), \\
-i \mathcal{M}_{A} & =\bar{u}\left(p_{1}^{\prime}\right)\left(i e_{3} \gamma^{\mu}\right) u\left(p_{1}\right)\left[\left\langle A_{\mu} A_{\nu}\right\rangle\right] \bar{u}\left(p_{2}^{\prime}\right)\left(i e_{3} \gamma^{\nu}\right) u\left(p_{2}\right),
\end{aligned}
$$

with $\langle\varphi \varphi\rangle$ and $\left\langle A_{\mu} A_{\nu}\right\rangle$ being the scalar and photon propagators. Expressions (6) and (7) represent the scattering amplitudes for electrons of equal polarization mediated by the scalar and gauge particles, respectively. The spinors $u(p)$ stand for the positive-energy solution of the Dirac equation $(\not p-m) u(p)=$ 0 . The $\gamma$ - matrices satisfy the so(1,2) algebra, $\left[\gamma^{\mu}, \gamma^{\nu}\right]=2 i \epsilon^{\mu \nu \alpha} \gamma_{\alpha}$, and correspond to the $(1+2)$ dimensional representation of the Dirac matrices, that is, the Pauli ones: $\gamma^{\mu}=\left(\sigma_{z},-i \sigma_{x}, i \sigma_{y}\right)$. Regarding these definitions, one obtains the spinors,

$$
u(p)=\frac{1}{\sqrt{N}}\left[\begin{array}{c}
E+m \\
-i p_{x}-p_{y}
\end{array}\right], \quad \bar{u}(p)=\frac{1}{\sqrt{N}}\left[\begin{array}{cc}
E+m & -i p_{x}+p_{y}
\end{array}\right],
$$

\footnotetext{
${ }^{1}$ We adopt a $1+2$-dimensional metric for space-time: $\eta_{\mu \nu}=(+,-,-)$.
} 
which fulfill the normalization condition $\bar{u}_{+}(p) u_{+}(p)=1$ whenever the constant $N=2 m(E+m)$ is adopted. The Möller scattering should be easily analyzed in the center of mass frame, where the momenta of the incoming and outgoing electrons are read at the form: $P_{1}^{\mu}=(E, p, 0), P_{2}^{\mu}=(E,-p, 0), P_{1}^{\prime \mu}=$ $(E, p \cos \theta, p \sin \theta), P_{2}^{\prime \mu}=(E,-p \cos \theta,-p \sin \theta)^{2}$. The transfer 4-momentum, carried by the gauge or scalar mediators, is: $k^{\mu}=P_{1}^{\mu}-P_{1}^{\prime \mu}=(0, p(1-\cos \theta),-p \sin \theta)$, whereas $\theta$ is the scattering angle (in the CM frame).

Considering the normalization condition satisfied by the spinors written in eq. (8), the scattering amplitude associated with the scalar sector can be readily evaluated,

$$
\mathcal{M}_{\text {scalar }}=y^{2} \frac{\left[k^{2}-s^{2}\right]}{\left[k^{4}-\left(s^{2}-v \cdot v\right) k^{2}-(v \cdot k)^{2}\right]} .
$$

which in the case of a purely timelike background, $v^{\mu}=\left(\mathrm{v}_{0}, \overrightarrow{0}\right)$, takes on the following form:

$$
\mathcal{M}_{\text {scalar }}=-y^{2} \frac{\left[\mathbf{k}^{2}+s^{2}\right]}{\mathbf{k}^{2}\left[\mathbf{k}^{2}+w^{2}\right]},
$$

where: $w^{2}=\left(s^{2}-\mathrm{v}_{0}^{2}\right)$, and it was used the general expression for the transfer momentum, $k^{\mu}=(0, \mathbf{k})$.

In connection with the gauge sector, only six terms of the gauge propagator contribute to the scattering amplitude $\left(\theta^{\mu \nu}, S^{\mu \nu}, \Lambda^{\mu \nu}, T^{\mu} T^{\nu}, Q^{\mu \nu}, Q^{\nu \mu}\right)$, as a consequence of the current-conservation law $\left(k_{\mu} J^{\mu}=0\right)$. The first two terms provide, in the non-relativistic limit, the Maxwell-Chern-Simons (MCS) scattering amplitude, already carried out in refs. [1]:

$$
\mathcal{M}_{M C S}=e^{2}\left\{\left(1-\frac{s}{m}\right) \frac{1}{\mathbf{k}^{2}+s^{2}}-\frac{2 s}{m} \frac{i \vec{k} \times \vec{p}}{\mathbf{k}^{2}\left(\mathbf{k}^{2}+s^{2}\right)}\right\}
$$

The total current-current amplitude mediated by the massive gauge particle corresponds to the sum of four contributions,

$$
\mathcal{M}_{\text {gauge }}=\mathcal{M}_{M C S}+\mathcal{M}_{\Lambda}+\mathcal{M}_{T T}+\mathcal{M}_{Q Q},
$$

where the terms $\mathcal{M}_{\Lambda}, \mathcal{M}_{T T}, \mathcal{M}_{Q Q}$ lead to background-depending corrections to the MCS-amplitude. To evaluate these three last terms, one first writes the following current-current amplitudes:

$$
\begin{aligned}
j^{\mu}\left(p_{1}\right)\left(T_{\mu} T_{\nu}\right) j^{\nu}\left(p_{2}\right) & =-2 \frac{p^{4}}{m} \mathrm{v}_{0}^{2} e^{i \theta}\left[1-\cos \theta+\sin ^{2} \theta\right] ; \\
j^{\mu}\left(p_{1}\right)\left(\Lambda_{\mu v}\right) j^{\nu}\left(p_{2}\right) & =\mathrm{v}_{0}^{2} ; \\
j^{\mu}\left(p_{1}\right)\left(Q_{\mu \nu}-Q_{\nu \mu}\right) j^{\nu}\left(p_{2}\right) & =2 \frac{p^{2}}{m} \mathrm{v}_{0}^{2}[1-\cos \theta-i \sin \theta] ;
\end{aligned}
$$

The first term does not contribute to the interaction potential as long as one works in the nonrelativistic approximation $\left(p^{2} \ll m^{2}\right)$. The other two terms lead to relevant contributions to the total amplitude scattering, namely:

$$
\mathcal{M}_{\Lambda}=-\frac{e^{2} s^{2} \mathbf{v}_{0}^{2}}{\mathbf{k}^{2}\left[\mathbf{k}^{2}+s^{2}\right]\left[\mathbf{k}^{2}+w^{2}\right]}, \mathcal{M}_{Q Q}=\frac{e^{2} s \mathrm{v}_{0}^{2}}{m} \frac{1}{\left[\mathbf{k}^{2}+s^{2}\right]\left[\mathbf{k}^{2}+w^{2}\right]}\left\{1-\frac{2 i \vec{k} \times \vec{p}}{\mathbf{k}^{2}}\right\},
$$

where $\vec{p}=\frac{1}{2}\left(\vec{p}_{1}-\vec{p}_{2}\right)$ is defined in terms of the momenta $\vec{p}_{1}, \vec{p}_{2}$ of the incoming electrons.

\footnotetext{
${ }^{2}$ Using this prescription and the 3-current definition, $j^{\mu}(p)=\bar{u}\left(p^{\prime}\right) \gamma^{\mu} u(p)$, the current components can be then explicitly written as: $j^{(0)}\left(p_{1}\right)=j^{(0)}\left(p_{2}\right)=\frac{1}{2 m(E+m)}\left[(E+m)^{2}+p^{2} e^{-i \theta}\right], j^{(1)}\left(p_{1}\right)=-j^{(1)}\left(p_{2}\right)=\frac{p}{2 m}\left(1+e^{i \theta}\right), j^{(2)}\left(p_{1}\right)=-j^{(2)}\left(p_{2}\right)=$ $\frac{i p}{2 m}\left(1-e^{i \theta}\right)$.
} 


\section{The electron-electron interaction potential}

\subsection{The scalar potential}

According to the Born approximation, the scalar interaction potential is given by the Fourier transform of the scattering amplitude (10), that is: $V_{\text {scalar }}(r)=\frac{1}{(2 \pi)^{2}} \int \mathcal{M}_{\text {scalar }} e^{i \vec{k} \cdot \vec{r}} d^{2} \vec{k}$. This integral can be exactly solved, resulting in the following expression:

$$
V_{\text {scalar }}(r)=-\frac{y^{2}}{(2 \pi)}\left\{\left[1+\frac{s^{2}}{w^{2}}\right] K_{0}(s r)-\frac{s^{2}}{w^{2}} \ln r\right\} .
$$

This potential reveals to be attractive near the origin and repulsive whenever the logarithmic term overcomes the Bessel-like one. Near the origin, the potential exhibits a genuine logarithmic behavior, once $K_{0}(x) \rightarrow-\ln x$ (for $x \rightarrow 0$ ). Far from the origin, the bessel function decays exponentially whereas the second term increases logarithmically. In (1+2)-dimensions, the logarithmic behavior is an outcome consistent with an unscreened interaction. Hence, the potential here obtained, at the level of a dynamical configuration, confirms the annihilation of the screening derived in ref. [7, at the level of a static evaluation. The result exhibited in eq.13) reflects the pole structure of the scalar amplitude, which possesses a massless $\left(1 / \mathbf{k}^{2}\right)$ and a massive pole $\left(1 /\left[\mathbf{k}^{2}+w^{2}\right]\right)$. The existence of the massless pole is ascribed to the fact the Chern-Simons pole $k^{2}=s^{2}$ to be deprived from dynamics [5].

\subsection{The gauge potential}

Carrying out the Fourier transform on the $\mathcal{M}_{M C S}$-amplitude, the corresponding Maxwell-Chern-Simons potential appears:

$$
V_{M C S}(r)=\frac{e^{2}}{(2 \pi)}\left[\left(1-\frac{s}{m}\right) K_{0}(s r)-\frac{2}{m s}\left[1-s r K_{1}(s r)\right] \frac{l}{r^{2}}\right],
$$

where $l=\vec{r} \times \vec{p}$ is the angular momentum (a scalar in a two-dimensional space).

The interaction potential associated with the amplitudes $\mathcal{M}_{\Lambda}, \mathcal{M}_{Q Q}$, can be also obtained from exact Fourier transform, resulting in the following expressions:

$$
\begin{gathered}
V_{\Lambda}(r)=\frac{e^{2}}{(2 \pi)}\left\{\frac{\mathrm{v}_{0}^{2}}{w^{2}} \ln r+\frac{s^{2}}{w^{2}} K_{0}(w r)-K_{0}(s r)\right\}, \\
V_{Q Q}(r)=\frac{e^{2}}{(2 \pi)}\left\{\frac{s}{m}\left[K_{0}(w r)-K_{0}(s r)\right]-\frac{2 s}{m} \frac{l}{r^{2}}\left[\frac{\mathrm{v}_{0}^{2}}{s^{2} w^{2}}-\frac{1}{w} r K_{1}(s r)+\frac{1}{s} r K_{1}(w r)\right]\right\} .
\end{gathered}
$$

The total gauge interaction potential, $V_{\text {gauge }}(r)=V_{M C S}+V_{\Lambda}+V_{Q Q}$, takes on the final form:

$$
\begin{aligned}
V_{\text {gauge }}(r) & =\frac{e^{2}}{(2 \pi)}\left\{-2(s / m) K_{0}(s r)+\left[s / m+s^{2} / w^{2}\right] K_{0}(w r)+\left(\mathrm{v}_{0}^{2} / w^{2}\right) \ln r\right. \\
& \left.-\frac{2}{m s} \frac{l}{r^{2}}\left[\left(1+\mathrm{v}_{0}^{2} / w^{2}\right)-\left(s^{2} / w\right) r K_{1}(s r)\right]\right\} .
\end{aligned}
$$

It is instructive to notice that one has $V_{\Lambda}, V_{Q Q} \rightarrow 0$ in the limit of a vanishing background $\left(\mathrm{v}_{0} \rightarrow 0\right)$, recovering the pure MCS result, given by eq. (14). Obviously, this is an expected outcome, since both $V_{\Lambda}, V_{Q Q}$ are potential contributions induced merely by the presence of the background. Taking the limit $r \rightarrow 0$ on the expression (17), one then determines the potential behavior near the origin, that is

$$
V_{\text {gauge }}(r) \simeq \frac{e^{2}}{(2 \pi)}\{C-(1-s / m-2 l s / m) \ln r\}
$$


where $C$ is a constant. Far from the origin, just the logarithmic term remains as dominant, so that:

$$
V_{\text {gauge }}(r) \simeq \frac{e^{2}}{(2 \pi)}\left[\frac{\mathrm{v}_{0}^{2}}{w^{2}}\right] \ln r
$$

Eqs. (18), (19) show that the gauge potential behaves logarithmically near and away from the origin, which puts again in evidence the annihilation of the screening [7, now manifest at the level of a dynamical evaluation. In the limit $r \rightarrow 0$, this potential may be attractive (for $s>m /(1+l)$ ) or repulsive (for $s<m /(1+l))$. In this paper one assumes $s^{2}>\mathrm{v}_{0}^{2}$, so that in the limit $r \rightarrow \infty$ the potential behaves repulsively. In the case $s>m /(1+l)$, there exists a region in which the potential is negative, a necessary premise for the formation of electron-electron bound states. For the case $s<m /(1+l)$, in which the potential is repulsive near and far the origin, just a graphical analysis can efficiently reveal the existence of a well (negative) region.

The real interaction corresponds to the total interaction potential, which comprises the gauge and the scalar contributions: $V(r)=V_{\text {scalar }}+V_{\text {gauge }}$. This total potential turns out attractive at the regions in which the negative scalar potential overcomes the repulsive gauge contribution, and at the regions for which the gauge potential is also negative. One then verifies that the total potential can always be negative at some region, which is a relevant result concerning the possibility of obtaining $e^{-} e^{-}$bound states in the framework of this particular model.

An important comparison to be made is allusive to the attractiveness of the gauge potential. In the case of the pure MCS potential, given by eq. (14), one must be careful in order to avoid a misleading interpretation of its low-energy behavior [1]. In such a regime, one must consider not only the centrifugal barrier term $\left(l^{2} / m r^{2}\right)$, but also the gauge invariant $A^{2}$-term coming from the Pauli equation,

$$
\left[\frac{(p-e A)^{2}}{m_{e}}+e \phi(r)-\frac{\vec{\sigma} \cdot \vec{B}}{m_{e}}\right] \Psi(r, \phi)=E \Psi(r, \phi),
$$

which rules the nonrelativistic behavior of a system in the presence of an electromagnetic field. The Laplacian operator, $\left[\frac{\partial^{2}}{\partial r^{2}}+\frac{1}{r} \frac{\partial}{\partial r}+\frac{1}{r^{2}} \frac{\partial^{2}}{\partial \phi^{2}}\right]$, corresponding to the $p^{2}$ term, acts on the total wavefunction $\Psi(r, \phi)=R_{n l}(r) e^{i \phi l}$, generating the repulsive centrifugal barrier term, $l^{2} /\left(m r^{2}\right)$. On the other hand, the $A^{2}$-term is essential to ensure the gauge invariance of a gauge mode in the nonrelativistic domain. This term does not appear in the context of a nonperturbative low-energy evaluation, for the same is associated with two-photon exchange processes (see Hagen and Dobroliubov [1). However, such a term must be suitably added up in order to assure the gauge invariance as well as to circumvent spurious behaviour concerning the low-energy potential.

In the presence of these two terms, the MCS potential reveals to be really repulsive instead of attractive. Hence, to correctly analyze the low-energy behavior of the gauge potential, it is necessary to add up the centrifugal barrier and the $A^{2}$ terms ${ }^{3}$ to the gauge potential previously obtained, leading to the following low-energy effective potential:

$$
V_{\text {eff }}(r)=V_{\text {gauge }}(r)+\frac{l^{2}}{m r^{2}}+\left(\frac{e}{2 \pi}\right)^{2}\left(\frac{s^{2}}{w^{2}}\right)^{2}\left[1-w r K_{1}(w r)\right]^{2} .
$$

The possibility for formation of electron-electron bound states is associated with the existence of a region in which the effective potential is negative. The figure below shows that this requirement is perfectly fulfilled for some parameters values:

\footnotetext{
${ }^{3}$ The vector potential, $A(r)$, was not determined in ref. 7], but it can be evaluated starting from the following coupled equations: $\nabla^{2}\left(\nabla^{2}-s^{2}\right) \vec{A}-\mathrm{v}_{0} \nabla^{2} \nabla^{*} \varphi=s \nabla^{*} \rho, \mathrm{v}_{0} \nabla \times A-\nabla^{2} \varphi=0$, derived in the static limit. The solution of these equations provides the required solution for the vector potential (in the case of pure a time-like background): $\vec{A}(r)=$ $\frac{e}{2 \pi} \frac{s^{2}}{w^{2}}\left[1-w r K_{1}(w r)\right] r^{*}$. In $(1+2)$ dimensions, the dual of a 2-vector is defined as $\left(E^{i}\right)^{*}=\epsilon_{i j} E^{j} \longrightarrow \vec{E}^{*}=\left(E_{y},-E_{x}\right)$, where one adopts the following convection: $\epsilon_{012}=\epsilon^{012}=\epsilon_{12}=\epsilon^{12}=1$.
} 


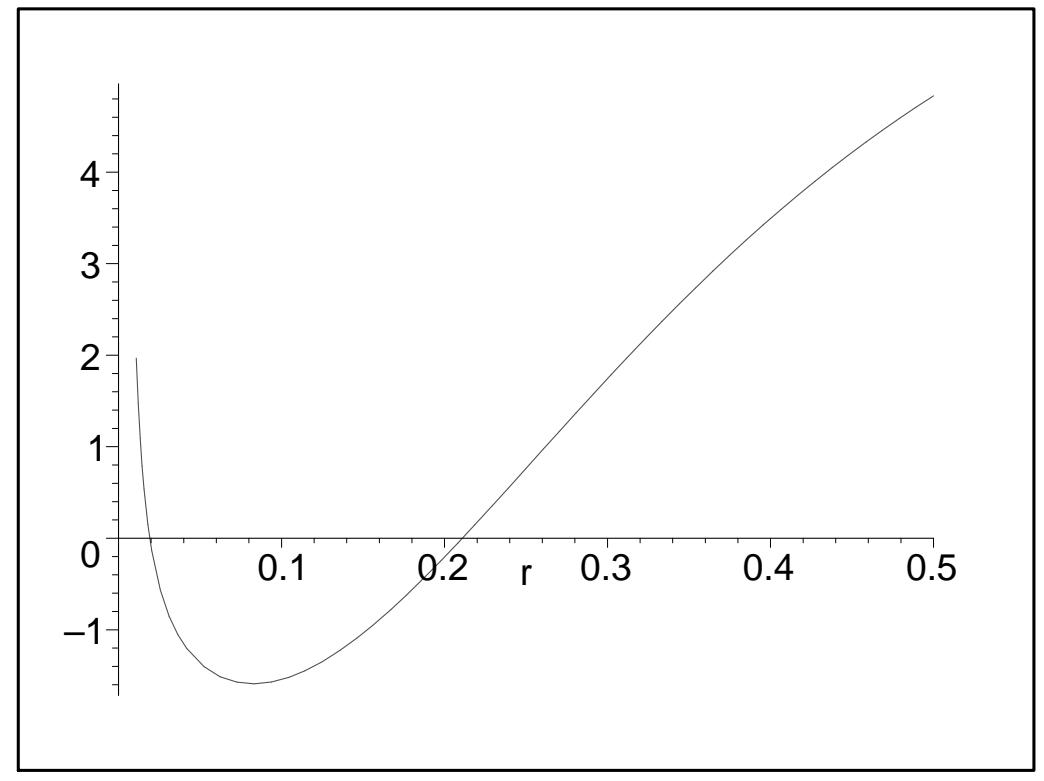

Figure 1: Effective potential for the following parameter values: $s=10, m=2000, \mathrm{v}_{0}=8, l=1$.

\section{Concluding Remarks}

In this work, one has considered the Möller scattering in the context of a planar Lorentz-violating MaxwellChern-Simons electrodynamics defined in a pure timelike background. The interaction potential was calculated as the Fourier transform of the scattering amplitude (Born approximation) carried out in the non-relativistic limit. The interaction potential exhibits two distinct contributions: the attractive scalar one (stemming form the Yukawa exchange) and the gauge one (mediated by the MCS-Proca gauge field). The scalar Yukawa interaction, as expected, turns out to be always negative. This makes feasible a global attractive potential, regardless the character (repulsive or attractive) of the gauge potential. In practice, such an interaction may be identified with phonon exchange processes, which represent physical excitations in several systems of interest. As for the gauge interaction, it is composed by a pure MCS potential corrected by background-depending contributions, which impose relevant physical modifications. The absence of screening, first observed in ref. [7, becomes now manifest in the context of a dynamical computation (by means of an ubiquitous logarithmic term), confirming the conclusion that $k^{2}=s^{2}$ is not a dynamical pole [5]. The background-depending corrections are such that they lead to an attractive gauge potential for some values of the parameters, which constitutes a promising result in connection with the possibility of obtaining the formation of Cooper pairs. This possibility can be appropriately checked up by means of a quantum-mechanical numerical analysis of the interaction potential here derived, which should be performed by means of the numerical solution of the Schrödinger equation. Such analysis must provide the corresponding $e^{-} e^{-}$binding energy once one takes suitable values for the parameters (in accordance with the scale of low-energy excitations typical in condensed matter systems).

A natural extension of this work consists in studying the interaction potential for the case of a purely spacelike background 11. Such an evaluation will certainly reveal an anisotropic potential in relation to the privileged direction fixed by the background, which may lead to an attractive interaction as well as an anisotropic $e^{-} e^{-}$order parameter.

In $(1+2)$ dimensions, the purely Coulombian interaction is associated with a logarithmic dependence, which implies in a confining rather than a binding behavior, which is ubiquitous in the results of this paper. However, the same can be eliminated if the gauge field exhibits an additional mass component, as the Proca term. Indeed, in a recent work [10] it was accomplished the dimensional reduction of an 
Abelian-Higgs Lorentz-violating model endowed with the CFJ term, resulting in a planar Maxwell-ChernSimons-Proca electrodynamics coupled to a massive Klein-Gordon field $(\varphi)$. A particular feature of this kind of Higgs model is the presence of totally screened modes: all its physical excitations are massive, which yields screened interactions. The consideration of the Möller scattering in this framework will lead to an entirely shielded interaction potential, once the logarithmic term should be suitably replaced by a $\mathrm{K}_{0}$ function.

Acknowledgement: The author is grateful to J. A. Helayël-Neto for reading and discussing this manuscript.

\section{References}

[1] H.O. Girotti et al., Phys. Rev. Lett. 69, 2623 (1992); C.R. Hagen, Phys. Rev. Lett. 71, 202 (1993); H.O. Girotti et al., Phys. Rev. Lett. 71, 203 (1993); M.I. Dobroliubov et al., Mod. Phys. Lett. A 8, 2177 (1993); A. Groshev and E.R. Poppitz, Phys. Lett. B 235, 336 (1990);Y. Georgelin and J.C. Wallet, Phys. Rev. D 50, 6610 (1994).

[2] H. Belich, O. M. Del Cima, M.M. Ferreira Jr. and J. A. Helayël-Neto, Int. J. Mod. Phys. A16, 4939 (2001); H. Christiansen, O. M. Del Cima. M.M. Ferreira Jr. and J. A. Helayël-Neto, Int. J. Mod. Phys. A18, 725 (2003); H. Belich et al., Eur. Phys. J. B 32, 145 (2003); -ibid, J. Phys. G 29, 1431 (2003).

[3] V. A. Kostelecky and S. Samuel, Phys. Rev. D 39,683 (1989); V. A. Kostelecky and R. Potting, Nucl. Phys.B 359, 545 (1991); ibid, Phys. Lett. B 381, 89 (1996); -ibid, Phys. Rev. D 51, 3923 (1995); C. Adam and F. R. Klinkhamer, Nucl. Phys. B 607, 247 (2001); C. Adam and F.R. Klinkhamer, Phys. Lett. B 513, 245 (2001); V.A. Kostelecky and R. Lehnert, Phys. Rev. D 63, 065008 (2001); A.A. Andrianov, P. Giacconi and R. Soldati, JHEP 0202, 030 (2002).

[4] D. Colladay and V. A. Kostelecký, Phys. Rev. D 55,6760 (1997); -ibid, Phys. Rev. D 58, 116002 (1998); S.R. Coleman and S.L. Glashow, Phys. Rev. D 59, 116008 (1999).

[5] H. Belich, M.M. Ferreira Jr., J. A. Helayël-Neto and M.T.D. Orlando, Phys. Rev. D 67, 125011 (2003); -ibid, Erratum to Phys. Rev. D 67, 125011 (2003).

[6] S. Carroll, G. Field and R. Jackiw, Phys. Rev. D 41, 1231 (1990);

[7] H. Belich, M.M. Ferreira Jr., J. A. Helayël-Neto and M.T.D. Orlando, Phys. Rev. D 68, 025005 (2003).

[8] W. N. Hardy et al., Phys. Rev. Lett. 70, 3999 (1993); Z.-X. Shen et al., Phys. Rev. Lett. 70, 1553 (1993); C.C. Tsuei et al., Phys. Rev. Lett. 72, 593 (1994); Phys. Today, january, 19 (1996); A.G. Sun et al., Phys. Rev. Lett. 72, 2267 (1994); K.A. Kouznetsov et al., Phys. Rev. Lett. 79, 3050 (1997).

[9] A. P. Baêta Scarpelli, H. Belich, J. L. Boldo and J. A. Helayël-Neto, Phys. Rev. D 67, 085021 (2003).

[10] H. Belich, M.M. Ferreira Jr., J. A. Helayël-Neto and M.T.D. Orlando, hep-th/0401235

[11] M. M. Ferreira Jr, work in progress.

[12] J. J. Sakurai, "Advanced Quantum Mechanics", Addison-Wesley Publishing Company, 1967. 